VIE SCIENTIFIQUE

CenTre Marc-Bloch

Schiffbauerdamm 19

D-10117 Berlin

Tél. + $493030874295 / 6$

Fax. +493030874301
Centre Franco-Allemand de Recherches en Sciences Sociales

Deutsch-Französisches Forschungszentrum für Sozialwissenschaften

\title{
ATELIER INTERNATIONAL
}

\section{Histoire comparée de la division du travail de dénombrement au $18^{e}$ siècle}

5 et 6 juin 1998

On connaît la grande diversité des dénombrements de population au $\mathrm{XVIII}^{e} \mathrm{~s}$. en Europe. Cet atelier a pour but de comparer les configurations de la division du travail savant et administratif qui les caractérisent dans chaque cas.

Il s'agira de comprendre les conditions de possibilite des travaux menés ici ou là, mais aussi celles de la réception des ouvrages produits ailleurs.

\author{
Vergleichende Geschichte \\ der numerischen \\ Arbeitsteilung \\ im 18. Jahrhunderts
}

\section{5.-6. Juni 1998}

Es ist bekannt, daB die Volkszăhlungen im 18. Jh. in Europa sehr unterschiedlich waren. Dieser Workshop hat zum Ziel, die Formen der Arbeitsteilung zwischen wissenschaftlich Arbeitenden und der Verwatlung zu vergleichen.

Es geht darum, die Entstehungsbedingungen von Arbeiten, die hier und da durchgeführt wurden, sowie die Art der Rezeption entsprechender ausländischer Werken zu verstehen.

\section{Comparative history of the division of numerical labor during the 18th Century}

June 5th-6th, 1998

The counting of population in 18th Century Europe displays a wide variety of configurations of the relationships between sciences and administrations. The workshop is intending to compare these various cases.

At stake is the comparative study of the preconditions of these works and of their reception further from their origins.

Organisé par Éric BRIAN avec le concours de l'EHESS (Paris)

Renseignements à demander à Laurence BEYER, Centre Marc Bloch (Berlin)

\section{Objectifs}

Comment concevait-on les dénombrements parmi les savants ou les administrateurs au $\mathrm{XVIII}^{\mathrm{e}}$ siècle, voire à la fin du XVII ${ }^{e}$ siècle ? Quelles formes de credit leur accordait-on ? Quelles genres légitimes gouvernaient ces pratiques et ces savoirs (la jurisprudence, la théologie, la médecine, les sciences) ? Dans quelles institutions étaient-ils l'objet de réflexions secrètes ou publiques? Par quelles voies les chiffres circulaient-ils (livres, sociétés savantes, correspondances aristocratiques et littéraires) ? Sous quelles formes ? L'atelier visera à répondre à ces questions en comparant des études de cas européens ou en ayant recours à des exemples coloniaux qui leur ont été contemporains. 
Centre MarC-Bloch Schiffbauerdamm 19 D-10117 Berlin

Tél. + $493030874295 / 6$

Fax. +493030874301
Centre Franco-Allemand de Recherches en Sciences Sociales

Deutsch-Französisches Forschungszentrum für Sozialwissenschaften

\section{ATELIER INTERNATIONAL}

\author{
Histoire comparée \\ de la division \\ du travail statistique \\ en Europe au 19 siècle
}

12 et 13 juin 1998

A l'issue d'une décennie de publications spécialisées sur l'histoire de la statistique au $\mathrm{XIX}^{\mathfrak{c}}$ siècle, cet atelier vise à établir des critères systématiques de comparaison des cas nationaux connus pendant la période de l'essor international de la production et du raisonnement statistiques (1840-1890).

\author{
Vergleichende Geschichte \\ der statistischen \\ Arbeitsteilung in Europa \\ des 19. Jahrhunderts
}

$$
\text { 12.-13. Juni } 1998
$$

Nach einem Jahrzehnt der Veröfentlichung von Werken zur Geschichte der Statistik im 19. Jh. ist das Ziel dieses Workshops, Kriterien für den Vergleich der statistischen Ämter in einzelnen Ländern wăhrend des internationalen Aufschwungs der Produktion von Statistiken und des statistischen Denkens zu entwickeln (1840-1890).

\section{Comparative history of the division of statistical labor in 19th Century Europe}

June 12th-13th, 1998

After a decade of publications on the history of statistics in the 19th Century, this workshop is an attempt to establish systematic comparative criteria upon various national cases for a period characterised by an international rise of statistical production and thinking (1840-1890).

\section{Organisé par Éric BRIAN et Morgane LABBÉ avec le concours de l'EHESS (Paris) Renseignements à demander à Laurence BEYER, Centre Marc Bloch (Berlin)}

\section{Objectifs}

Il s'agit de comparer les modalités concrètes du travail statistique, les formes de légitimité dans lesquelles elles étaient prises (académies, universités, gouvernements, pouvoirs législatifs). Les réseaux des protagonistes, des institutions, les voies de circulation des ouvrages et des savoir-faire seront encore scrutés. Une attention particulière sera portée, pays par pays, à la périodisation de la transformation des activités statistiques. On situera enfin les échanges entre statisticiens dans le contexte de la tenue des neufs sessions du Congrès international de statistique (1853-1876). On s'interrogera sur les différents niveaux auxquels on peut observer les transformations étudiées - local, municipal, national, international, central ou périphérique-, échelles elles-mêmes soumises à des renouvellements importants pendant la même période. 